\title{
Chronic Lymphocytic Leukemia and Autoimmune Cytopenias: Pathogenesis, Diagnosis and Treatment
}

\author{
Fergun Yilmaz ${ }^{*}$, Demet Kiper ${ }^{1}$, Filiz Vural ${ }^{2}$ \\ ${ }^{1}$ Izmir Katip Celebi University, Ataturk Training and Research Hospital, Karabaglar, Izmir, Turkey \\ ${ }^{2}$ Ege University Medical School, Bornova, Izmir, Turkey
}

Received: May 11, 2017; Accepted: May 20, 2017; Published: May 29, 2017

*Corresponding author: Fergun Yilmaz, MD, Izmir Katip Celebi University, Ataturk Training and Research Hospital Izmir Turkey, E-mail:fergunaydin@ hotmail.com

\begin{abstract}
Chronic Lymphocytic Leukemia (CLL) is a clonal proliferation of B cells in bone marrow, lymph nodes, peripheral blood, spleen and other lymphoid tissues. CLL is mostly associated with dysregulated immune system. Intrinsic immune dysfunction results in increased frequency of autoimmune disorders and susceptibility to infections at the time of diagnosis or during course of the disease. Autoimmune cytopenias especially Autoimmune Hemolytic Anemia (AIHA) is observed frequently. AIHA is the most common immune cytopenia ranging between $5-10 \%$ and it is followed by less frequent Immune Thrombocytopenia (IT) (1-5\%). Autoimmune Neutropenia (AIN) and Pure Red Cell Aplasia (PRCA) are rare entities $(<1 \%)$. In CLL patients, the differential diagnosis of autoimmune cytopenias is important since there are many confounding factors which may cause cytopenia in CLL patients such as infiltration of bone marrow by malignant cells, chemotherapy and infections. A careful diagnostic workup should be performed including history taking, physical examination, laboratory tests and bone marrow aspiration and biopsy. The treatment of AIHA and IT is similar to their counter parts primary AIHA and IT where the steroids are the treatment of choice. In PRCA, steroids are mostly ineffective and immunosuppressive agents should be tried. AIN is a very rare entity in CLL patients. Granulocyte stimulating factors, immunosuppressive therapies may be options in symptomatic patients.
\end{abstract}

\section{Introduction}

Chronic Lymphocytic Leukemia (CLL) is a clonal proliferation of B cells in bone marrow, lymph nodes, peripheral blood, spleen and all other lymphoid tissues. The B cells have a distinctive immunophenotype characterized by CD5, CD19, CD20 and CD23 positivity [1,2]. Among other leukemias, CLL is mostly associated with dysregulated immune system. Intrinsic immune dysfunction results in increased frequency of autoimmune disorders and susceptibility to infections at the time of diagnosis or during the course of the disease. Quantitative and qualitative dysfunction may be observed in both humoral and cellular immune system. Defects in B cells may result in poor response to vaccinations and hypogammaglobulinemia. Defects in T cells, natural killer cells, macrophages, complement system and neutrophils can also be detected. All these alterations are responsible for increased incidence of infections in these patients even in the absence of chemotherapy. Therapy induced immunosuppression also add additional risks [3-7]. Bacterial lower respiratory tract infections with $S$. pneumonia, $S$ aureus and $H$. influenza are most commonly observed infections in these patients. Viral infections including H. simplex and H. zoster are also documented in CLL patients $[6,8,9]$. Particular attention should be given to the treatment and prophylaxis of these infections to decrease the mortality and morbidity. The risk factors, type of infections, treatment and prophylaxis approaches and associated impairment in immunity is beyond the scope of this review.

Other spectrum in dysfunctional immunity in CLL patients is autoimmunity. Both hematologic and non-hematologic autoimmune disorders can be observed. The incidence of secondary malignancies is also increased in these patients, another entity related to altered immune system. Autoimmune cytopenias especially Autoimmune Hemolytic Anemia (AIHA) are most common autoimmune diseases. The rest of the article will be focused on autoimmune cytopenias.

\section{Epidemiology of autoimmune disorders}

Association of autoimmune diseases with CLL was recognized more than fifty years ago [10]. In a study including 2017 patients, it was reported that although cytopenias in CLL patients may be due to many factors such as bone marrow infiltration, chemotherapy or splenomegaly, autoimmune cytopenias may contribute a considerable number of cases [11]. AIHA is the most common immune cytopenia ranging between 5-10\% that is followed by less frequent Idiopathic Thrombocytopenia (IT) (1-5 \%). Autoimmune Neutropenia (AIN) and Pure Red Cell Aplasia (PRCA) are rare entities ( $<1 \%$ ) [11-15]. The prevalence of AIHA is related to stage of the disease and the presence or absence of the IGVH mutation. Although the prevalence of AIHA in early stage disease is approximately $3 \%$, nearly $10 \%$ of CLL patients in Binet $\mathrm{B}$ and $\mathrm{C}$ may develop AIHA. Also, the prevalence is high in patients with unmutated IGVH [6].

\section{Pathogenesis}

The interaction between CLL cells and autoimmunity is complex. Many possible mechanisms and hypothesis try to explain the pathogenesis. In some autoimmune diseases such 
as cold agglutinin and paraneoplastic pemfigus, CLL cell itself may secrete autoantibodies that react with specific self-antigen. In paraneoplastic pemfigus, the self-antigens are at the dermal epidermal junction and in cold agglutinin, $M$ type antibodies react with red blood cell antigens. CLL cells may also process the antigens and act as aberrant antigen presenting cells. After interaction with $\mathrm{T}$ cells, normal $\mathrm{B}$ cells will be activated to produce polyclonal antibodies against red blood cell antigens [16]. Hall et al also agreed that malignant cells might drive an autoimmune response by acting as antigen presenting cells [17]. Another possible mechanism is that cytokines produced by malignant clone may cause loss of tolerance to self-antigens and increase the possibility of escape of self-reactive clones. Autoantigens may also activate malignant B cells $[12,15,16]$. None of these mechanisms alone may explain the underlying pathogenesis in all autoimmune diseases.

Both $\mathrm{T}$ and $\mathrm{B}$ cells are involved in the pathogenesis of autoimmune disorders. Thrombocytopenia and AIHA are mostly associated with polyclonal immunoglobulins (Ig) mainly Ig G type directed against antigens on erythrocytes and thrombocytes, respectively $[15,17]$. When these autoantibodies are reacted with antigens on erythroblasts in the bone marrow or neutrophils, this will result in PRCA and AIN, respectively $[15,18]$. The exact source of these antibodies is not well defined. $\mathrm{B}$ cells belonging to malignant clone or normal B cells activated by several possible mechanisms may produce autoantibodies. It is thought that resting normal B cells may be activated to produce auto-antibodies against antigens on normal blood cells including erythrocytes, neutrophils or platelets via $\mathrm{T}$ cell dysfunction and abnormal interaction between $\mathrm{T}$ and $\mathrm{B}$ cells $[15,19]$. Regulatory $\mathrm{T}$ cells ( $\mathrm{T}$ reg) also plan an important role in pathogenesis in development of autoimmune diseases in CLL patients. Their abnormal expression or dysregulation in CLL patients will lead to escape of self-reactive cells that cause autoimmune diseases $[15,16,20,21]$.

Autoimmune diseases in CLL patients may be observed during chemotherapy especially with fludarabine. But fludarabine related AIHA will not be discussed in this review.

\section{Autoimmune cytopenias and prognosis}

In the literature, there are conflicting data about the effect of autoimmune cytopenias on prognosis. It is not well defined if patients with autoimmune cytopenias have a poor prognosis or not. First of all, autoimmune cytopenias should be differentiated from cytopenias due to bone marrow infiltrations since patients with cytopenia due to bone marrow infiltrations, namely anemia and thrombocytopenia are classified as advanced staged disease according to Rai and Binet staging and have poorer prognosis [22,23]. Moreno et al analyzed 960 CLL patients. Autoimmune cytopenia was documented in $7 \%$ of these patients at the time of diagnosis, before diagnosis or during the course of the disease. Patients with autoimmune cytopenia had higher lymphocyte count, higher expression of ZAP 70 and CD 38 and rapid blood lymphocyte doubling time but outcome of these patients was not significantly different when compared to those without autoimmune cytopenia. But it was evident in this study that patients with cytopenia due to bone marrow involvement had a worse prognosis than patients with cytopenia due to autoimmunity [24]. This data confirmed by another study by Zent et al. They evaluated 1750 patients with CLL. They reported that survival was significantly better in patients with autoimmune cytopenias when compared to patients with cytopenias related to bone marrow infiltration. The median survival was 9.1 years in patients with autoimmune cytopenia and 4.4 years in patients with cytopenias due to bone marrow infiltration $(\mathrm{p}<0.001)$. In this study, although cytopenia with bone marrow involvement was associated with poor prognosis, this was not demonstrated in patients with autoimmune cytopenias [11]. On the other hand, Visco et al, suggested that autoimmune cytopenias at the diagnosis of CLL might have a negative effect on prognosis [25]. In another study, Direct Antiglobulin Test ( DAT ) positivity with or without AIHA and AIHA were found to be associated with reduced overall survival [26]. Visco et al, retrospectively analyzed 1278 newly diagnosed CLL patients. In this large study, unmutated IGVH, a positive DAT test, and occurrence of AIHA was associated with development of IT significantly. They also demonstrated a poorer prognosis in CLL patients with IT [27].

Autoimmune cytopenias are also associated with welldefined clinical and biologic prognostic factors such as advanced stage disease, age, high number of white cells, unmutated IGVH, short lymphocyte doubling time [11,16, 24, 28, 29]. The impact of these factors on the effect of autoimmune cytopenias on prognosis should be clarified.

Since there is conflicting data in the literature related to prognostic significance of autoimmune cytopenias, long term follow up studies are needed to resolve this question.

\section{Diagnosis}

\section{AIHA}

AIHA should be suspected in patients with CLL when there is an unexpected fall in hemoglobin level. This fall will be gradual or sudden and the presentation of the patient is based on the rate of development of anemia. As in idiopathic AIHA, high Lactate Dehydrogenase (LDH) and indirect bilirubin levels, reticulocytosis, reduced haptoglobin and positive DAT test is the hallmark for the diagnosis. Peripheral blood smear may reveal spherocytes and polychromasia. Contrast to idiopathic AIHA, in CLL patients high LDH levels may be due to active disease or reticulocytosis may not be obvious because of bone marrow infiltration or anemia because of PRCA. So, careful work up should be performed to rule out other reasons of anemia in CLL patients. Bone marrow aspiration and biopsy should be the part of this work up. DAT test has a special consideration in CLL patients. DAT positivity is not always associated with hemolysis and anemia. It may be observed alone without any evidence of hemolysis. The incidence of pretreatment DAT positivity was $14 \%$ in the UK LRF CLL4 trial [26]. In addition, all the patients with DAT positivity did not develop AIHA. It was also demonstrated that DAT positivity was associated with poorer outcome $[6,12,26]$. So , in the presence of DAT positivity accompanying 
anemia and hemolysis must be documented before the diagnosis of AIHA. On the other hand, DAT negative AIHA may also be observed. Ig A type autoantibodies or low affinity antibodies may explain the negative results. In addition, the amount the auto-antibodies are also important in evaluating the DAT results. When the amount of antibody is below the threshold level of the test, these antibodies will not be detected and the test will reveal negative results although the autoantibodies are present. More sensitive tests or anti IgA antibodies may resolve these problems but these tests could not be performed in all laboratories in routine practice [13]. DAT positivity in CLL patients is not always related to autoantibodies. Especially in pretransfused CLL patients, hemolysis due to alloantibodies, delayed type hemolytic transfusion reaction, should be also ruled out to confirm the diagnosis as DAT positive AIHA.

Evans syndrome should also be kept in mind in CLL patients with AIHA although Evans syndrome is rare than AIHA $[24,30,31]$.

Essential diagnostic workup is summarized in [Table 1]

\begin{tabular}{|c|c|}
\hline Test & Comment* \\
\hline \multirow{2}{*}{$\begin{array}{l}\text { Complete blood } \\
\text { count }\end{array}$} & Anemia should be documented. \\
\hline & Rule out other reasons \\
\hline $\begin{array}{l}\text { Peripheral blood } \\
\text { smear }\end{array}$ & Spherocytosis (not always detected) \\
\hline Reticulocyte & $\begin{array}{l}\text { Reticulocytosis predict AIHA } \\
\text { (May be low in PRCA or bone marrow } \\
\text { involvement) }\end{array}$ \\
\hline Haptoglobulin & $\begin{array}{l}\text { May be increased due to underlying } \\
\text { inflammatory processes }\end{array}$ \\
\hline $\begin{array}{l}\text { Biochemical } \\
\text { parameters } \\
\text { including LDH, } \\
\text { bilirubin }\end{array}$ & $\begin{array}{l}\text { High levels (high LDH levels may also be } \\
\text { associated with underlying progressive } \\
\text { disease or liver disease. Indirect bilirubin } \\
\text { levels may be normal if the liver is intact.) }\end{array}$ \\
\hline DAT test & $\begin{array}{l}\text { DAT test positivity is the hallmark of the } \\
\text { disease. But negative test does not rule out } \\
\text { AIHA and positive test is not always associated } \\
\text { with AIHA }\end{array}$ \\
\hline $\begin{array}{l}\text { Bone marrow } \\
\text { aspiration and } \\
\text { biopsy }\end{array}$ & $\begin{array}{l}\text { Massive infiltration, lack of erythroid } \\
\text { precursors may explain the reason for anemia }\end{array}$ \\
\hline
\end{tabular}

\section{IT}

IT is a less common complication than AIHA in CLL patients. Bleeding is not associated even with very low platelet counts. It may be due to splenomegaly and hipersplenism, bone marrow infiltration, myelotoxic effects of chemotherapy.
Although thrombocytopenia due to bone marrow involvement is mostly observed in late stages, IT may occur at any stage. Since there is not a routine test similar to DAT used in AIHA, the diagnosis of CLL associated secondary IT is more complicated. Some criteria are postulated to make the diagnosis [Table 2] $[13,27]$. First of all, the number of the thrombocytes should be decreased more than $50 \%$ from the basal level and the absolute number should be less than $100.000 / \mu$ l. The number of the thrombocytes should be confirmed with peripheral blood smear to rule out pseudo-thrombocytopenia and macro thrombocytes. The decrease in platelet number should not be explained by another reason such as chemotherapy or splenomegaly and hipersplenism. Other reasons causing thrombocytopenia such as infections, disseminated intravascular coagulation, Human Immunodeficiency Virus (HIV), hepatitis C, and drug induced thrombocytopenia should be ruled out by appropriate tests [13,32-34]. Table 3 demonstrates some of the possible causes of thrombocytopenia in CLL patients. Bone marrow biopsy or aspiration should reveal normal or increased number of megakaryocytes due to reactive pattern. Demonstrating anti platelet antibodies is not recommended routinely in CLL patients. First of all, these antibodies are not sensitive and specific and secondly, they are not routinely performed in most of the laboratories.

\begin{tabular}{|c|c|}
\hline Criteria & Explanation \\
\hline $\begin{array}{l}\text { 1. Decrease in number of } \\
\text { platelets }\end{array}$ & $\begin{array}{l}>50 \% \text { from the baseline and } \\
\qquad<100.000 / \mathrm{ml}\end{array}$ \\
\hline 2. No splenomegaly & $\begin{array}{l}\text { Limited splenomegaly that can not } \\
\text { be palpated }\end{array}$ \\
\hline $\begin{array}{l}\text { 3. Normal or elevated } \\
\text { megakaryocytes } \\
\text { in the bone marrow }\end{array}$ & - \\
\hline $\begin{array}{l}\text { 4. No chemotherapy related } \\
\text { thrombocytopenia }\end{array}$ & - \\
\hline $\begin{array}{l}\text { 5. Exclusion of other } \\
\text { possible causes }\end{array}$ & $\begin{array}{c}\text { Recent chemotherapy } \\
\text { Other autoimmune secondary } \\
\text { cytopenias } \\
\text { related to infections such as HIV, } \\
\text { hepatitis } \\
\text { or drug induced }\end{array}$ \\
\hline
\end{tabular}

The diagnosis of CLL associated IT is sometimes so difficult to confirm. Under these circumstances, after all the possible causes are excluded by a careful history (including drugs and clues for recent infections) and laboratory tests, a trial of Intravenous Immunoglobulin (IVIG) and steroid or response to platelet suspensions may help to confirm the diagnosis $[13,31]$. 
Rapid response to IVIG or steroid will be a clue for autoimmune cytopenia. Refractoriness to platelet transfusion may also support the diagnosis of IT. When there is isolated thrombocytopenia in a CLL patient, this is favor of IT since anemia mostly accompanies thrombocytopenia in patients with bone marrow involvement. But this is not true in the case of Evans syndrome in which IT and anemia coexist. So, essential attention should be given to evaluate patients presenting with both anemia and thrombocytopenia to make the correct diagnosis.

In daily practice, rapid decrease in platelet count without evidence of bone marrow involvement and other possible causes, supports an autoimmune nature. A trial of intravascular immunoglobulin and/or steroid will confirm the diagnosis [27]

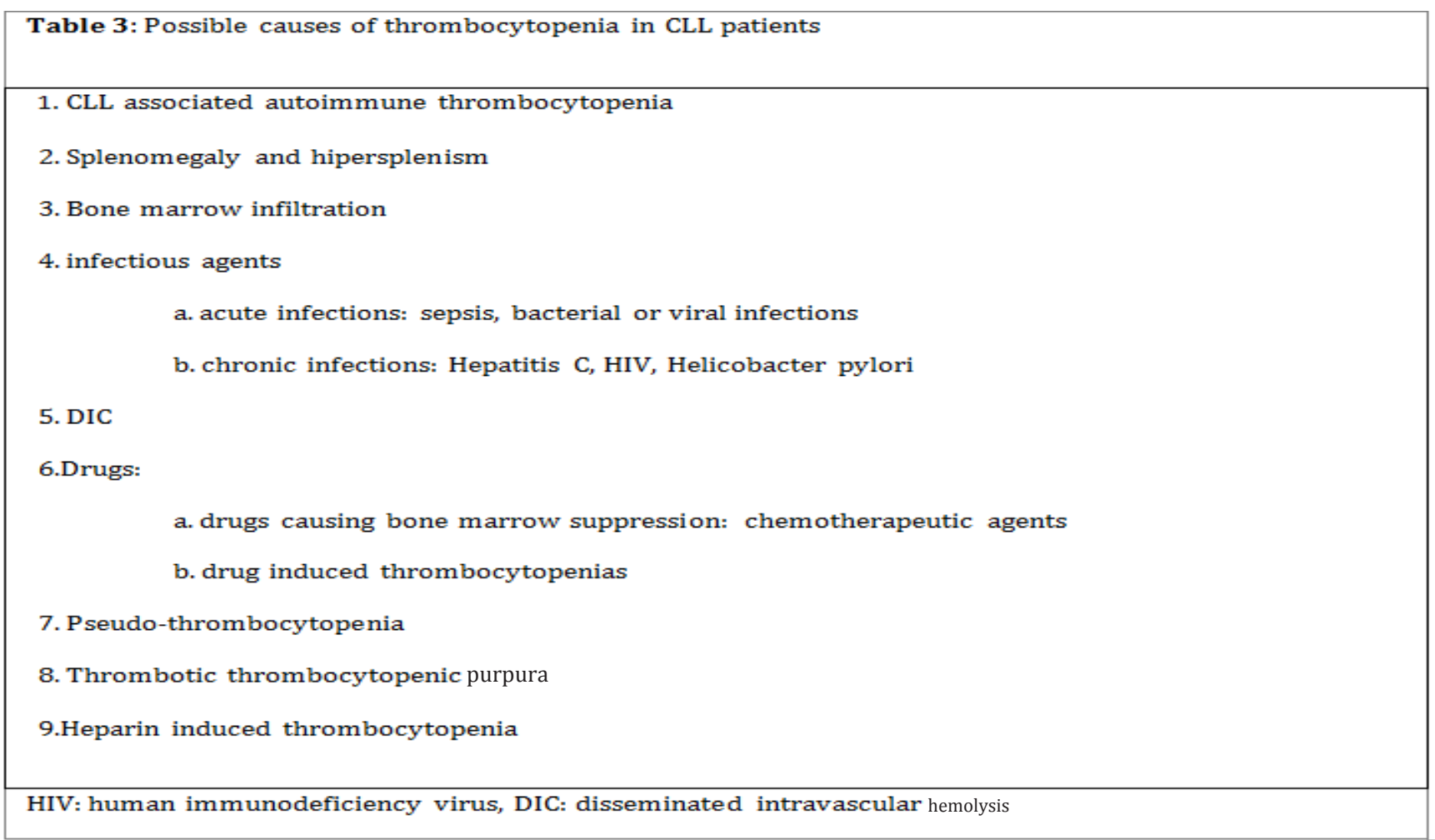

\section{PRCA}

PRCA is a rare entity in CLL patients. The recommended criteria for the diagnosis includes anemia, reticulocytopenia and absence of erythroid precursors in the bone marrow in a patient with CLL without an obvious cause [13,18,31]. Vitamin B12 and folic acid levels should be checked especially in patients with macrocytosis [12]. Other causes especially viral infections should be ruled out. In a case series, $\mathrm{Xu}$ et al reported seven patients with PRCA related to CytoMegaloVirus (CMV) and Epstein - Barr virus (EBV). The diagnosis was confirmed by Polymerase Chain Reaction (PCR) for CMV infection and Ig M antibodies against EBV capsid antigen and PCR for EBV infection [35]. A Parvovirus B19 infection should also be ruled out since its association with PRCA is demonstrated in the literature [36]. Other possible associated viral infections are HIV or hepatitis B and C. Bone marrow biopsy and aspiration should be performed to document the absence of erythroid precursors in the marrow [Table 4].

Table 4: Criteria for the diagnosis of PRCA

\begin{tabular}{|l|l|}
\hline Anemia & $\begin{array}{l}\text { Normochromic normocytic } \\
\text { Can not be explained by other causes } \\
\text { Hemoglobin }<11 \mathrm{gr} / \mathrm{dl}\end{array}$ \\
\hline Absolute reticulocytopenia & $\begin{array}{l}\text { Be careful that reticulocytopenia may be } \\
\text { associated with bone marrow infiltration } \\
\text { in a patient with anemia and bone marrow infiltration }\end{array}$ \\
\hline Bone marrow biopsy and aspiration & $\begin{array}{l}<1 \% \text { of bone marrow cells } \\
\text { Granulocytic and megakaryocytic lines } \\
\text { should be relatively normal }\end{array}$ \\
\hline
\end{tabular}


Rule out other possible causes

\author{
No recent chemotherapy \\ No hemolysis \\ DAT negativity \\ No parvovirus infection \\ Normal LDH and bilirubin levels
}

DAT: Direct antiglobulin test, LDH: lactate dehydrogenase,

AIN

It is also a rare entity in CLL patients. Neutropenia without any other cause is the hallmark of the disease. Antineutrophil antibodies can be detected in serum of the patients but negative results cannot be ruled out of the AIN. In addition, presence of the auto-antibodies against neutrophil antigens is not always associated with the clinical scenario [12]. The diagnosis is mainly based on exclusion of all other causes [29]. The long-term bone marrow suppression effect of chemotherapeutic agents should be taken into consideration in the differential diagnosis because more than one year of neutropenia may be observed after fludarabine- cyclophosphamide - rituximab combination [37]. Bone marrow aspiration and biopsy should be performed to rule out other reasons such as myelodysplastic features and infiltration by malignant cells [29].

\section{Treatment}

There are no randomized controlled trials for the treatment of autoimmune cytopenias related to CLL so decision to treat should be personalized. In the case of absence of progressive disease, the treatment modalities are similar to the treatment of primary counterparts [38,39].

\section{AIHA}

In the treatment of AIHA, the first step is to decide if transfusion is mandatory or not. Hemoglobin levels, signs and symptoms of the patient and patient characteristics such as comorbidities, age should be taken into account for the decision of transfusion. Due to the presence of autoantibodies against red blood cell antigens, compatible cross match is nearly impossible and incompatible cross matched erythrocyte transfusion is not contraindicated under selected circumstances [38,39]. In relatively emergent cases in whom rapid increase in hemoglobin level is preferred, intravenous immunoglobulin (IVIG) can be given.

First line therapy is similar to patients with primary AIHA. If warm antibody is the cause of hemolysis, prednisone 1 $\mathrm{mg} / \mathrm{kg} /$ day is the preferred regimen. The starting dose can be continued up to 3-4 weeks. Afterwards, the dose should be tapered slowly within 1-2 months. Alternatively high dose dexamethasone ( $40 \mathrm{mg} /$ day) for four days can be used as first line therapy when the patient is not suitable for long term steroid therapy [13]. But there is not enough data for its use in CLL associated AIHA. Especially during prolonged usage of steroids treatment with vitamin $\mathrm{D}$ and calcium preparations should be remembered. Gastro-protective therapy, folic acid supplementation and observation for steroid induced diabetes should be kept in mind in all patients. It is important to differentiate warm AIHA from cold agglutinin disease because cold agglutinin disease is mostly refractory to steroids and rituximab $(375 \mathrm{mg} / \mathrm{m} 2 /$ day $)$ weekly for four weeks is the preferred regimen $[6,13]$.

In refractory patients and patients who need high dose maintenance steroid therapy, second line treatments should be considered. Rituximab is a good option in refractory AIHA patients $[40,41]$. The overall response (complete remission and partial remission) rate is as high as $70 \%$ with a durable transfusion free period [43]. The therapy scheme for rituximab is of $375 \mathrm{mg} / \mathrm{m} 2$ weekly for four weeks [13]. Although lower doses, a total of $100 \mathrm{mg}$ weekly for four weeks, were effective and safe in primary AIHA, its role in CLL related AIHA should be clarified [42,43]. Rituximab plus chemotherapy is also an alternative regimen in steroid refractory patients [40]. Several combinations of rituximab with cytotoxic agents were studied in small number of patients. Rituximab plus Cyclophosphamide plus Dexamethasone (RCD) is one of the options [44,45]. RCD revealed an overall response rate as high as $89.5 \%$ with a 24 months' median duration of response [45]. Good response rates were also reported by a combination regimen of rituximab, cyclophosphamide, vincristine and prednisone (RCVP) [46]. Rituximab bendamustine (BR) combination was studied in a multicentric retrospective study by Quinquenel et al. The overall response rate was $81 \%$ and median time to next treatment was 28.3 months. It was concluded that BR was a safe and effective therapy in CLL patients with refractory AIHA [47]. Other monoclonal antibodies such as ofatumumab or alemtuzumab may be tried but there is very limited data about their use in CLL related AIHA [13]. Splenectomy in eligible patients should also be kept in mind [6].

In refractory patients, cyclophosphamide and other immunosuppressive agents such as cyclosporine, mycophenolate mofetil, or azathioprine may be used. Although safety and efficacy data is lacking, these agents may be tried in refractory patients based on limited data on patients with primary AIHA [13,48-50].

\section{IT}

Patients with IT are treated when the platelet count is below $30.000 / \mu$ l or thrombocytopenia is associated with bleeding. Similar to AIHA, prednisone therapy $1 \mathrm{mg} / \mathrm{kg} /$ day is recommended for a period of 3-4 weeks. Afterwards, gradual tapering of the dose is required. IVIG can be considered in patients with bleeding or prior to surgery. In refractory patients, rituximab $375 \mathrm{mg} / \mathrm{m} 2$ weekly for four weeks is an alternative $[29,40]$. D'Arena et al evaluated the efficacy and safety of rituximab monotherapy in 21 patients with CLL associated 
IT. The infusion side effect was observed in only one patient. Complete and partial rates were $57 \%$ and $29 \%$ respectively with a mean duration of response of 21 months [51]. Similar to AIHA, rituximab in combination with cytotoxic agents such as RCD or RCVP can be considered in eligible patients [40,4446]. Splenectomy is not always suitable in this elderly patient group. In addition, it increases the risk of infections. Under these circumstances, splenectomy can be performed inhighly-selected patients.

Another option in refractory patients is thrombopoietin mimetics. Although there is only small case series evaluating its activity in CLL associated IT, it should be considered in suitable patients $[12,52]$.

\section{PRCA}

The treatment of PRCA is somewhat different from AIHA and IT since it does not respond well to steroids. In the case of PRCA, cyclosporine is the treatment of choice [53,54]. Long term therapy is required so side effects such as hypertension, renal insufficiency, immune suppression and hyperlipidemia should be carefully followed especially in elderly patients. IVIG ( $400 \mathrm{mg} / \mathrm{kg} /$ day for 5 days) may be an alternative especially in patients with PRCA associated with Parvovirus 19 infection. The alternative effective therapies include rituximab alone or in combination with dexamethasone and cyclophosphamide [40].

\section{AIN}

Since it is a rare entity, there is very limited data about the treatment options and efficacy. Spontaneous remissions may be observed. Immunosuppressive therapies, steroids or rituximab and granulocyte stimulating factors may be tried in symptomatic patients $[13,40]$.

In conclusion, CLL patients may experience autoimmune cytopenias at the time of diagnosis or during the course of the disease. A careful work up should be carried out to make the right diagnosis and to choose the appropriate treatment. Since large prospective studies are lacking in the literature, the treatment approach should be personalized. New targeted therapies and new monoclonal antibodies should be studied in especially refractory patients.

\section{References}

1. Swerdlow S, International Agency for Research on C, World Health O. WHO classification of tumours of haematopoietic and lymphoid tissues. Lyon France: International Agency for Research on Cancer 4th ed. 2008.

2. Hallek M, Cheson BD, Catovsky D, et al. Guidelines for the diagnosis and treatment of chronic lymphocytic leukemia: a report from the International Workshop on Chronic Lymphocytic Leukemia updating the National Cancer Institute-Working Group 1996 guidelines. Blood. 2008;111(12):5446-5456.

3. Tsiodras S, Samonis G, Keating MJ, Kontoyiannis DP. Infection and immunity in chronic lymphocytic leukemia.Mayo Clin Proc. 2000;75(10):1039-1054.

4. Wadhwa PD, Morrison VA. Infectious complications of chronic lymphocytic leukemia. Semin Oncol. 2006;33(2):240-249. doi: 10.1053/j.seminoncol.2005.12.013
5. Strati P, Shanafelt TD. Monoclonal B-cell lymphocytosis and earlystage chronic lymphocytic leukemia: diagnosis, natural history, and risk stratification. Blood. 2015;126(4):454-462. doi: 10.1182/ blood-2015-02-585059

6. Dearden C. Disease-specific complications of chronic lymphocytic leukemia. Hematology Am Soc Hematol Educ Program. 2008:450-456. doi: 10.1182/asheducation-2008.1.450

7. Scrivener S, Goddard RV, Kaminski ER, Prentice AG. Abnormal T cell function in B-cell chronic lymphocytic leukaemia. Leuk Lymphoma. 2003;44:383-389.

8. Morrison VA. Infectious complications in patients with chronic lymphocytic leukemia : pathogenesis, spectrum of infection, and approaches to prophylaxis. Clin Lymphoma Myeloma. 2009;9(5):365370. doi:10.3816/CLM.2009.n.071

9. Morrison VA. Management of infectious complications in patients with chronic lymphocytic leukemia. Hematology Am Soc Hematol Educ Program. 2007:332-338.

10. Ebbe S, Wittels B, Dameshek W. Autoimmune thrombocytopenic purpura ("ITP" type) with chronic lymphocytic leukemia. Blood. 1962;19:23-37

11.Zent CS, Ding W, Schwager SM, Megan S. Reinalda, James D. Hoyer, et al. The prognostic significance of cytopenia in chronic lymphocytic leukemia/small lymphocytic lymphoma (CLL). Br J Haematol. 2008;141(5):615-621.

12. Hodgson K, Ferrer G, Pereira A, Moreno C, Montserrat E. Autoimmune cytopenia in chronic lymphocytic leukaemia: diagnosis and treatment. Br J Haematol. 2011;154(1):14-22. doi: 10.1111/j.13652141.2011.08707.x

13. Visco C, Barcellini W, Maura F, Neri A, Cortelezzi A, Rodeghiero F. Autoimmunecytopenias in chronic lymphocytic leukemia. Am J Hematol. 2014;89(11):1055-1062. doi: 10.1002/ajh.23785

14.Zent CS, Kay NE. Autoimmune complications in chronic lymphocytic leukaemia (CLL). Best Pract Res Clin Haematol. 2010;23(1):47-59. doi: 10.1016/j.beha.2010.01.004

15. D’Arena G, Guariglia R, La RoccaF, et al. Autoimmune Cytopenias in Chronic Lymphocytic Leukemia. Clinical and Developmental Immunology. 2013 (2013). doi: 10.1155/2013/730131

16. Hodgson KFG, Montserrat E, Moreno C. Chronic lymphocytic leukemia and autoimmunity: a systematic review. Haematologica. 2011;96(5):752-761. doi: 10.3324/haematol.2010.036152

17. Hall AM, Vickers MA, McLeod E, Barker RN. Rh autoantigen presentation to helper $\mathrm{T}$ cells in chronic lymphocytic leukemia by malignant B cells. Blood. 2005;105(5):2007-2015.

18. D’Arena G, Cascavilla N. Chronic lymphocytic leukemia-associated pure red cell aplasia. Int J Immunopathol Pharmacol. 2009;22(2):279286.

19. Hamblin TJ. "Autoimmune Complications of Chronic Lymphocytic Leukemia," Seminars in Oncology. 2006;33(2):230-239.

20. Riches JC, Ramsay AG, Gribben JG. T-cell function in chronic lymphocytic leukemia. Semin Cancer Biol. 2010;20:431-438. doi: 10.1016/j.semcancer.2010.09.006

21. Lad DP, Varma S, Varma N, Sachdeva MU, Bose P, Malhotra P. Regulatory T-cell and T-helper 17 balance in chronic lymphocytic leukemia progression and autoimmune cytopenias. Leuk Lymphoma. 2015;56(8):2424-2428.

22. Rai KR, Sawitsky A, Cronkite EP, et al. Clinical staging of chronic lymphocytic leukemia. Blood 1975;46:219-234.

23. Binet JL, Auquier A, Dighiero G, et al. A new prognostic classification of chronic lymphocytic leukemia derived from a multivariate survival 
analysis. Cancer. 1981;48(1):198-206.

24. Moreno C, Hodgson K, Ferrer G, Elena M, Filella X, Pereira A, et al. Autoimmune cytopenias in chronic lymphocytic leukemia: prevalence, clinical associations, and prognostic significance. Blood. 2010;116(23):4771-4776.

25. Visco C, Cortelezzi A, Moretta F, Falisi E, Maura F, Finotto S, et al. Autoimmune cytopenias in chronic lymphocytic leukemia at disease presentation in the modern treatment era: is stage $\mathrm{C}$ always stage $\mathrm{C}$ ? Leuk Lymphoma. 2014;55(6):1261-1265.

26. Dearden C, Wade R, Else M, Richards S, Milligan D, Hamblin T, et al. The prognostic significance of a positive direct antiglobulin test in chronic lymphocytic leukemia: a beneficial effect of the combination of fludarabine and cyclophosphamide on the incidence of hemolytic anemia. Blood. 2008;111(4):1820-1826.

27. Visco C, Ruggeri M, Laura Evangelista M, Stasi R, Zanotti R, Giaretta I, et al. Impact of immune thrombocytopenia on the clinical course of chronic lymphocytic leukemia. Blood. 2008;111(3):1110-1116.

28. Mauro FR, Foa R, Cerretti R, Giannarelli D, Coluzzi S, Mandelli F, et al. Autoimmune hemolytic anemia in chronic lymphocytic leukemia: clinical, therapeutic, and prognostic features. Blood. 2000;95(9):27862792

29. Tsang M, Parikh SA. A Concise Review of Autoimmune Cytopenias in Chronic Lymphocytic Leukemia. Curr Hematol Malig Rep 2017;12(1):29-38. doi: 10.1007/s11899-017-0366-1

30. Evans RS, Takahashi K, Duane RT, Payne R, Liu C. Primary thrombocytopenic purpura and acquired hemolytic anemia; evidence for a common etiology. AMA Arch Intern Med. 1951;87(1):48-65.

31. Tandra P, Krishnamurthy J, Bhatt VR, et al. Autoimmune Cytopenias in Chronic Lymphocytic Leukemia, Facts and Myths. Mediterr J Hematol Infect Dis. 2013;5(1):e2013068.

32. Neunert C, Lim W, Crowther M, et al. The American Society of Hematology 2011 evidencebased practice guideline for immune thrombocytopenia. Blood. 2011;117:4190-4207. doi: 10.1182/ blood-2010-08-302984

33. Dahal S, Upadhyay S, Banjade R, Dhakal P, Khanal N, Bhatt VR. Thrombocytopenia in Patients with Chronic Hepatitis C Virus Infection. Mediterr J Hematol Infect Dis. 2017;9(1):e2017019. doi 10.4084/MJHID.2017.019

34.Tsang M, Parikh SA. A Concise Review of Autoimmune Cytopenias in Chronic Lymphocytic Leukemia. Curr Hematol Malig Rep. 2017;12(1):29-38. doi: 10.1007/s11899-017-0366-1

35. Xu L, Fang JP, Weng WJ, et al. Pure red cell aplasia associated with cytomegalovirus and Epstein-Barr virus infection in seven cases of Chinese children. Hematology. 2013;18(1):56-59.

36. Crabol Y, Terrier B, Rozenberg F, Pestre V, Legendre C, Hermine O, et al. Intravenous immunoglobulin therapy for pure red cell aplasia related to human parvovirus b 19 infection: a retrospective study of 10 patients and review of the literature. Clin Infect Dis. 2013;56(7):968977. doi: $10.1093 /$ cid/cis1046

37. Fischer K, Bahlo J, Fink AM, Goede V, Herling CD, Cramer P, et al. Long-term remissions after FCR chemoimmunotherapy in previously untreated patients with CLL : updated results of the CLL8 trial. Blood. 2016;127(2):208-215. doi: 10.1182/blood-2015-06-651125

38. Yilmaz F, Vural F. Autoimmune Hemolytic Anemia:Focusing on Therapy According to Classification. SOJ Immunol 2017;4(1):1-6. doi: 10.15226/2372-0948/4/1/00156

39. Lechner K, Jäger U. How I treat autoimmune hemolytic anemias in adults. Blood. 2010;116(11):1831-1838. doi:10.1182/ blood-2010-03-259325

40. Laurenti L, Vannata B, Innocenti I, et al. The use of monoclonal antibodies in the treatment of autoimmune complications of chronic lymphocytic leukemia. Mediterr J Hematol Infect Dis. 2013;5(1):e2013027.

doi: 10.4084/MJHID.2013.027

41. D'Arena G, Laurenti L, Capalbo S, et al. Rituximab therapy for chronic lymphocytic leukemia-associated autoimmune hemolytic anemia. Am J Hematol 2006;81:598-602.

42. Fu R, Yan S, Wang X, Wang G, Qu W, Wang H, et al. A monocentric retrospective study comparing pulse cyclophosphamide therapy versus low dose rituximab in the treatment of refractory autoimmune hemolytic anemia in adults. Int J Hematol. 2016;104(4):462-467. doi: 10.1007/s12185-016-2056-5

43. Barcellini W, Zaja F, Zaninoni A et al. Sustained response to lowdose rituximab in idiopathic auto immune hemolytic anemia. 2013;91(6):546-551. doi: 10.1111/ejh.12199

44. Gupta N, Kavuru S, Patel D, et al. Rituximab-based chemotherapy for steroid-refractory autoimmune hemolytic anemia of chronic lymphocytic leukemia. Leukemia. 2002;16(10):2092-2095. doi: 10.1038/sj.leu.2402676

45. Rossignol J, Michallet AS, Oberic L, et al. Rituximab-cyclophosphamidedexamethasone combination in the management of autoimmune cytopenias associated with chronic lymphocytic leukemia. Leukemia. 2011;25:473-478. doi: 10.1038/leu.2010.278

46. Bowen DA, Call TG, Shanafelt TD, et al. Treatment of autoimmune cytopenia complicating progressive chronic lymphocytic leukemia/ small lymphocytic lymphoma with rituximab, cyclophosphamide, vincristine, and prednisone. Leukemia and Lymphoma. 2010;51:620627. doi: $10.3109 / 10428191003682767$

47. Quinquenel A, Willekens C, Dupuis J, et al. Bendamustine and rituximab combination in the management of chronic lymphocytic leukemia-associated autoimmune hemolytic anemia: a multicentric retrospective study of the French CLL intergroup (GCFLLC/MW and GOELAMS). Am J Hematol. 2015;90(3):204-207.

48. Kotb R, Pinganaud C, Trichet C, et al. Efficacy of mycophenolate mofetil in adult refractory auto-immune cytopenias: a single center preliminary study. Eur J Haematol. 2005;75(1):60-64

49. Emilia G, Messora C, Longo G, Bertesi M. Long-term salvage treatment by cyclosporin in refractory auto immune haematological disorders. Br J Haematol. 1996;93(2):341-344.

50. Lechner K, Jäger U How I treat autoimmune hemolytic anemias in adults. Blood. 2010;116(11):1831-1838. doi:10.1182/ blood-2010-03-259325

51. D’Arena G, Capalbo S, Laurenti L, et al. Chronic lymphocytic leukemiaassociated immune thrombocytopenia treated with rituximab: a retrospective study of 21 patients. Eur J Haematol. 2010;85(6):502507. doi: 10.1111/j.1600-0609.2010.01527.x

52. Jain N, Keating MJ, Burger JA, et al. A phaseII trial of eltrombopag for patients with chronic lymphocytic leukemia (CLL) and thrombocytopenia. Blood. 2013;122:4186.

53. Chikkappa G, Pasquale D, Zarrabi MH, Weiler RJ, Divakara M, Tsan MF. Cyclosporine and prednisone therapy for pure red cell aplasia in patients with chronic lymphocytic leukemia. Am J Hematol. 1992;41(1):5-12.

54. Means RT Jr. Pure red cell aplasia. Blood. 2016;128(21):2504-2509. 